\title{
NRROS Negatively Regulates Osteoclast Differentiation by Inhibiting RANKL-Mediated NF- $\kappa B$ and Reactive Oxygen Species Pathways
}

\author{
Jung Ha Kim, Kabsun Kim, Inyoung Kim, Semun Seong, and Nacksung Kim*
}

\begin{abstract}
Negative regulator of reactive oxygen species (NRROS) is known to repress ROS generation in phagocytes. In this study, we examined the roles of NRROS in both osteoclasts and osteoblasts. Our results demonstrate that NRROS negatively regulates the differentiation of osteoclasts, but not osteoblasts. Further, overexpression of NRROS in osteoclast precursor cells attenuates RANKLinduced osteoclast differentiation. Conversely, osteoclast differentiation is enhanced upon siRNA-mediated knockdown of NRROS. Additionally, NRROS attenuates RANKLinduced NF-KB activation, as well as degradation of the NOX1 and NOX2 proteins, which are required for ROS generation. Based on our observations, we present NRROS as a novel negative regulator of RANKL-induced osteoclastogenesis.
\end{abstract}

\section{INTRODUCTION}

Bone homeostasis is maintained by a delicate balance between osteoblast and osteoclast functions (Rho et al., 2004). Osteoblasts, which are responsible for bone formation, differentiate from the mesenchymal lineage under the control of bone morphogenetic factor 2 (BMP2) signaling cascades (Kamiya, 2012). Meanwhile, osteoclasts, which are responsible for bone resorption, differentiate from mononuclear progenitors of the macrophage lineage in the presence of macrophage colony stimulating factor (M-CSF) and receptor activator of NF-KB ligand (RANKL) (Walsh et al., 2006).

Notably, RANKL is a cytokine essential for osteoclast differentiation. Binding of RANKL to its receptor, RANK, induces the expression of transcription factors including c-Fos and NFATc1, and activates early signaling pathways including mitogenactivated protein kinases (MAPKs) and NF- $\kappa B$. RANKL induces c-Fos at the early stages of osteoclast differentiation, which in turn induces NFATc1 expression. NFATc1, a master transcrip-

Department of Pharmacology, Medical Research Center for Gene Regulation, Chonnam National University Medical School, Gwangju 501-746, Korea

*Correspondence: nacksung@jnu.ac.kr

Received 19 June, 2015; revised 13 August, 2015; accepted 13 August, 2015; published online 7 October, 2015

Keywords: NRROS, osteoclast differentiation, RANKL tion factor for osteoclast differentiation, subsequently induces the expressions of osteoclastic genes such as tartrate-resistant acid phosphatase (TRAP), cathepsin $\mathrm{K}$, dendritic cell-specific transmembrane protein (DC-STAMP), and osteoclast-associated receptor (OSCAR), which are essential to osteoclast differentiation and function (Kim and Kim, 2014; Kim et al., 2005; 2008; Walsh et al., 2006). The early signaling pathways activated by RANKL include NF- $\kappa B$, Akt, Jun N-terminal kinase (JNK), p38 kinase, and extracellular signaling related kinase (ERK). Previously, several studies have shown that the activation of these signaling pathways also plays a key role in osteoclast differentiation and function (Boyle et al., 2003; He et al., 2011; Moon et al., 2012). Furthermore, RANKL generates reactive oxygen species (ROS) in osteoclasts and their precursors to promote their differentiation and resorption (Lee et al., 2005; Sasaki et al., 2009).

Recently, Noubade et al. described a protein that they termed negative regulator of ROS (NRROS) (Noubade et al., 2014). NRROS localizes to the endoplasmic reticulum (ER), where it negatively regulates ROS generation during inflammatory response. The authors showed that NRROS facilitates ROS repression through degradation of the NADPH oxidase 2 (NOX2), dependent on the ER-associated degradation pathway (Noubade et al., 2014).

In this study, we investigated the roles of NRROS in both osteoclasts and osteoblasts. We observed that while NRROS has no direct effect on the differentiation and function of osteoblasts, it negatively regulates osteoclast differentiation. Upon probing the underlying mechanism, we found that it does so by inhibiting the RANKL-mediated NF-кB pathway and by promoting the degradation of both NOX1 and NOX2.

\section{MATERIAL AND METHODS}

\section{Reagents}

Recombinant human M-CSF was a gift from Dr. Daved Fremont (Washington University, USA), while recombinant human SRANKL was purified from bacteria. Recombinant human BMP2 was purchased from Cowellmedi (Korea). Alizarin red and $\beta$-glycerophosphate were obtained from Sigma-Aldrich (USA). Ascorbic acid was purchased from Junsei Chemical (Japan). While antibodies specific to FLAG and HA tags were purchased from Sigma-Aldrich, those against phospho-p38, p38, phospho-JNK, JNK, phospho-Erk, Erk, and IKB were obtained from Cell Signaling Technology (USA). 


\section{Osteoclast differentiation}

Murine osteoclasts were prepared from bone marrow cells as previously described (Kim et al., 2007; 2015). In brief, mouse bone marrow cells were isolated from tibiae and femurs of 6-8 week old ICR mice by flushing the bone marrows with $\alpha$ minimal essential medium ( $\alpha-M E M)$. Bone marrow cells were cultured in $\alpha$-MEM (Hyclone Laboratories, USA), containing $10 \%$ fetal bovine serum (FBS) with M-CSF (30 ng/ml), for 3 days. Adherent cells [bone marrow-derived macrophage-like cells (BMMs)] were further cultured with M-CSF (30 ng/ml) and RANKL (20-150 ng/ml) for 3 days. Cultured cells were fixed and stained for TRAP. TRAP-positive cells with more than three nuclei were counted as osteoclasts.

\section{Osteoblast differentiation}

Primary osteoblasts were isolated from neonatal mouse calvaria by successive enzymatic digestion with $0.1 \%$ collagenase (Life Technologies) and 0.2\% dispase II (Roche Diagnostics $\mathrm{GmbH}$, Germany). Osteoblasts were cultured in osteogenic medium containing BMP2 (100 ng/ml), ascorbic acid $(50 \mu \mathrm{g} / \mathrm{ml})$, and $\beta$-glycerophosphate $(100 \mathrm{mM})$. To assess their differentiation, osteoblasts cultured for 3 days were stained for alkaline phosphatase (ALP). To assess ALP activity, cells were lysed in osteoblast lysis buffer $[50 \mathrm{mM}$ Tris- $\mathrm{HCl}(\mathrm{pH} 7.4), 1 \%$ Triton $\mathrm{X}$ $100,150 \mathrm{mM} \mathrm{NaCl}$, and $1 \mathrm{mM}$ EDTA], and the lysates were incubated with p-nitrophenyl phosphate substrate (SigmaAldrich). ALP activity was then measured at an absorbance of $405 \mathrm{~nm}$. To assess their function, osteoblasts cultured for 9 days were fixed with $70 \%$ ethanol and stained with $40 \mathrm{mM}$ alizarin red $(\mathrm{pH} 4.2)$. For alizarin red solution assay, stained alizarin red was dissolved with $10 \%$ cetylpyridinium (Sigma) and the extracted solution was then measured at an absorbance of $562 \mathrm{~nm}$.

\section{Retroviral gene transduction}

Retroviral vectors were transfected into the packaging cell line, Plat E, using FuGENE 6 (Promega) according to the manufacturer's instruction. Viral supernatants were harvested from the culture medium 48 hours after transfection. BMMs or osteoblasts were then incubated with the viral supernatants for $6 \mathrm{~h}$ in the presence of polybrene (Sigma-Aldrich).

\section{Quantitative Real-Time PCR}

The cultured cells were lysed in Qiazol lysis reagent (Qiagen, Germany) for total RNA extraction. Purified RNA was then reverse transcribed into cDNA using Superscript II Reverse Transcriptase (Life Technologies) according to the manufacturer's protocol. Quantitative Real-Time PCR was performed in triplicate using SYBR mixture (Qiagen). The relative mRNA amounts were then calculated by normalizing to the endogenous housekeeping gene, GAPDH.

\section{Western blot analysis}

The cultured cells were lysed in extraction buffer [50 mM Tris$\mathrm{HCl}$ (pH 8.0), $150 \mathrm{mM} \mathrm{NaCl}, 1 \mathrm{mM}$ EDTA, 0.5\% Nonidet P-40, and protease inhibitors). Cell lysates were then subjected to sodium dodecyl sulfate polyacrylamide gel electrophoresis (SDS PAGE), and transferred electrophoretically onto a PVDF
$\boldsymbol{A}$

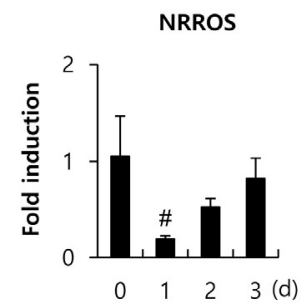

TRAP

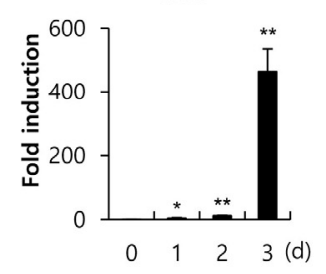

c-Fos

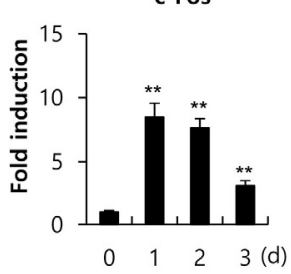

OSCAR

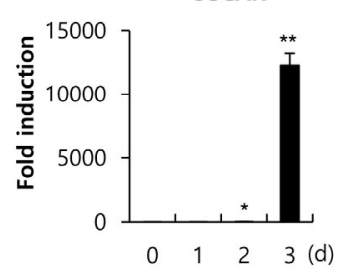

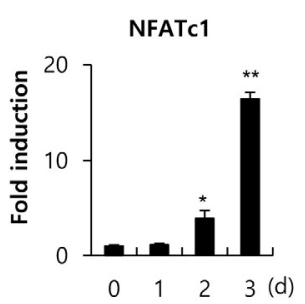

B

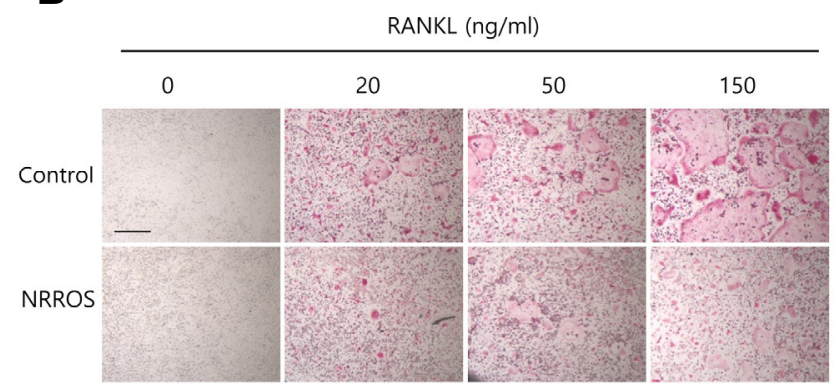

$\square$ Control $\square$ NRROS

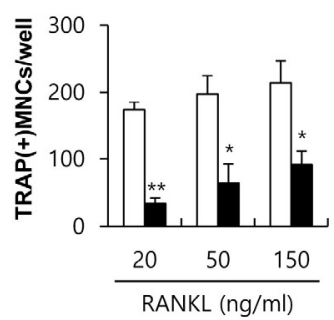

Fig. 1. Negative regulator of reactive oxygen species (NRROS) inhibits receptor activator of NF- $\mathrm{kB}$ ligand (RANKL)-induced osteoclastogenesis. (A) Bone marrow-derived macrophage cells (BMMs) were cultured with macrophage colony-stimulating factor (M-CSF) and RANKL for the time-periods indicated. mRNA levels of NRROS, cFOS, NFATC1, ACP5 (TRAP), and OSCAR were assessed by quantitative Real-Time PCR. Data represent the mean \pm standard deviation (SD) of samples in triplicates. \#, * and ** indicate $p$ values $<0.05,<0.01$ and $<0.001$, respectively, versus (vs.) the 0 day. (B) BMMs were transduced with either pMX-IRES-EGFP (control) or NRROS retrovirus, and then cultured in the presence of M-CSF and various concentrations of RANKL for 3 days. The cultured cells were stained for TRAP (left panel). The numbers of TRAP+ multinucleate cells (MNCs) per well were counted (right panel). ${ }^{*}$ and ${ }^{* *}$ indicate $p$ values $<0.01$ and $<0.001$, respectively, vs the control. Bar, $100 \mu \mathrm{m}$. 
A

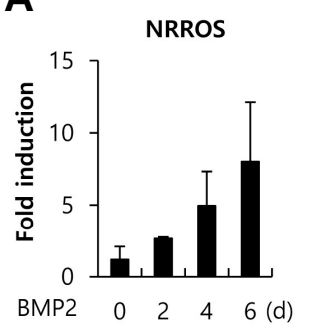

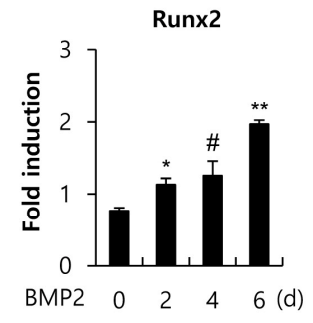

B

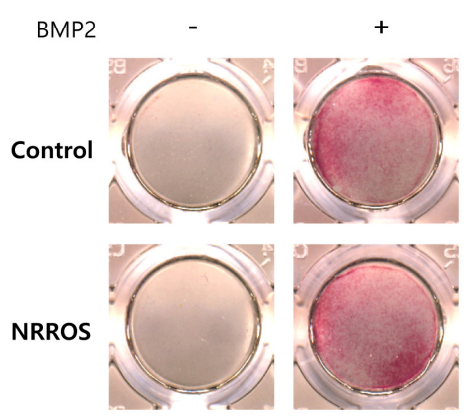

C

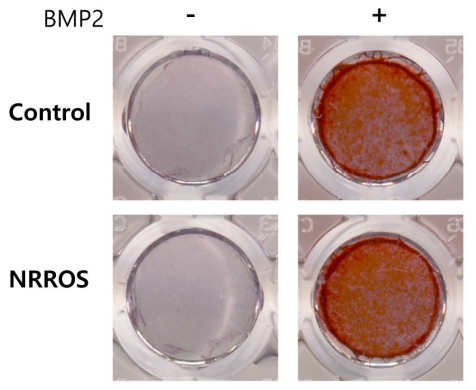

ALP

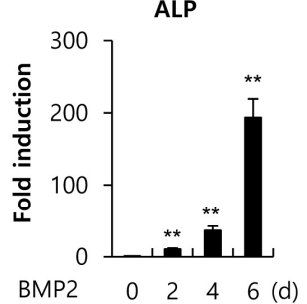

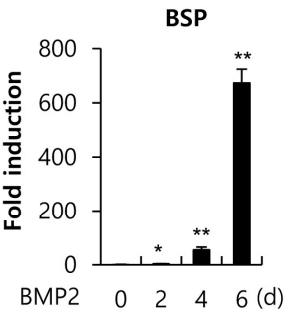

Fig. 2. NRROS does not affect osteoblast differentiation and function. (A) Primary osteoblasts were cultured in osteogenic medium (OGM) containing BMP2, ascorbic acid, and $\beta$ glycerophosphate for the timeperiods indicated. mRNA levels of NRROS, RUNX2, ALPL(ALP), and $I B S P(B S P)$ were assessed by quantitative Real-Time PCR. Data represent the mean \pm SD of samples in triplicates. \#, * and ${ }^{* *}$ indicate $p$ values $<0.05$, $<0.01$, and $<0.001$, respectively, vs. the 0 day. $(B, C)$ Osteoblasts were transduced with either pMX-IRES-EGFP (control) or NRROS retrovirus and cultured in OGM. (B) Cells cultured for 4 days were fixed and stained for alkaline phosphatase (ALP) (left panel). ALP activities were measured by densitometry at $405 \mathrm{~nm}$ (right panel). (C) Cells cultured for 9 days were fixed and stained for alizarin red (left panel), which was quantified by densitometry at $562 \mathrm{~nm}$ (right panel).

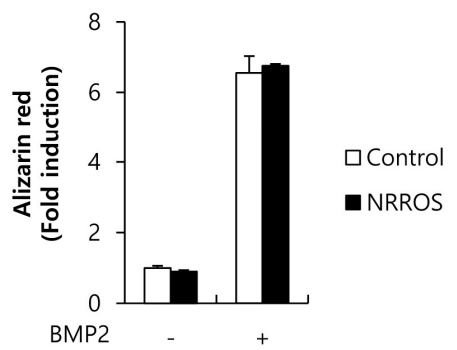

membrane (Millipore). The membranes were subjected to western blot analysis and signals were detected by a LAS3000 luminescent image analyzer (GE Healthcare Japan, Japan).

\section{Measurement of intracellular ROS}

Cultured cells were washed with PBS and then incubated with $10 \mu \mathrm{M} 2^{\prime}, 7^{\prime}$-dichlorodihydrofluorescein-diacetate (DCFH-DA) for $30 \mathrm{~min}$. 2',7'-dichlorofluorescein (DCF) fluorescence was measured with an excitation wavelength of $488 \mathrm{~nm}$ and emission at 515-540 nm.

\section{Statistical analysis}

All data are expressed as the mean \pm SD. Statistical analyses were performed using the unpaired Students' $t$-test. $p$ values less than 0.05 were considered statistically significant.

\section{RESULTS}

\section{Overexpression of NRROS inhibits RANKL-induced osteo-} clast differentiation

We first examined whether NRROS is expressed in osteoclast lineage cells using Real-Time PCR. BMMs were cultured in the presence of M-CSF and RANKL for 3 days to induce osteoclast differentiation. During the RANKL-mediated osteoclast differen- tiation, expressions of osteoclastic genes such as c-Fos, NFATc1, TRAP, and OSCAR were significantly increased (Fig. 1A). NRROS was expressed in the BMMs to a lesser extent on day 1 , but gradually heightened on days 2 and 3 during osteoclast differentiation (Fig. 1A). In order to elucidate the role of NRROS in osteoclasts, we overexpressed NRROS in BMMs by retroviral transduction. As shown in Fig. 1B, RANKL strongly induced osteoclast differentiation in the control vector-infected BMMs. Interestingly, NRROS overexpression in BMMs significantly attenuated the formation of TRAP-positive multinucleate cells (MNCs) compared to that in control cells. These results together indicate that NRROS is a negative regulator of RANKL-mediated osteoclast differentiation.

\section{NRROS overexpression does not affect osteoblast differentiation and function}

Next, we examined the expression patterns and function of NRROS during osteoblast differentiation. Primary osteoblasts were cultured in osteogenic medium containing BMP2, ascorbic acid, and $\beta$-glycerophosphate. We observed that the expressions of osteoblast-associated genes such as RUNX2, ALP, and $B S P$ increased as the cells differentiated (Fig. 2A). The expression of NRROS was also increased during osteoblast differentiation (Fig. 2A). Next, to investigate the effect of NRROS on oste- 
A

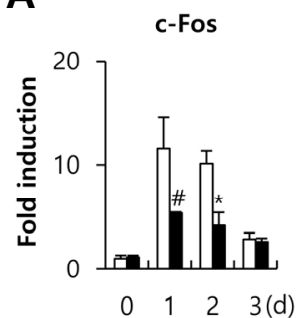

$\square$ Control $\square$ NRROS
NFATc1

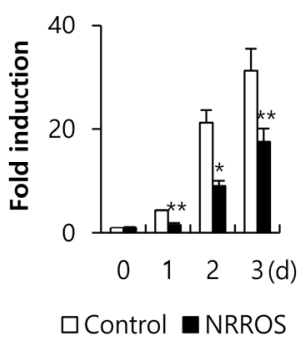

TRAP

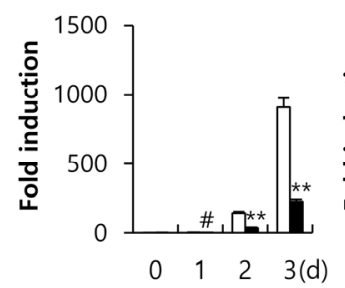

$\square$ Control anRROS
OSCAR

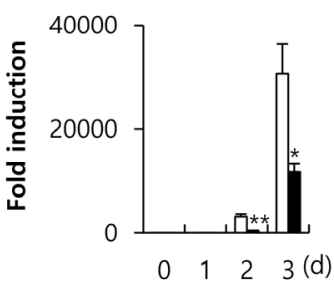

口Control anRROS

B

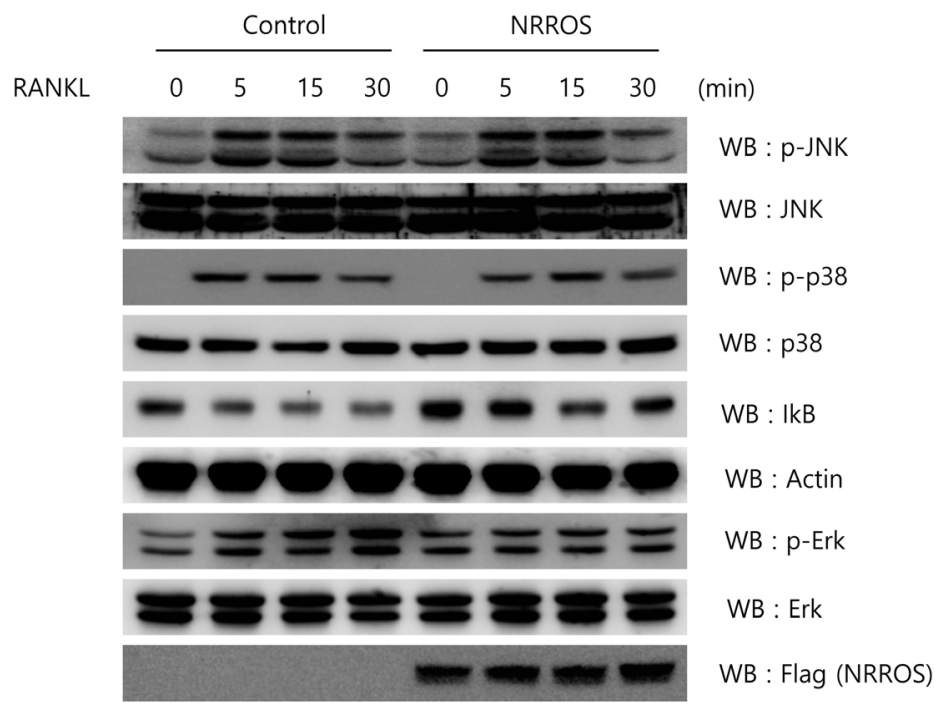

Fig. 3. Effects of NRROS on RANKL-induced gene expressions and signaling pathways. (A) BMMs were transduced with either pMX-IRESEGFP (control) or NRROS retrovirus, and then cultured in the presence of M-CSF and RANKL for the indicated time-periods. mRNA levels of $c$-FOS, NFATC1, ACP5(TRAP), and OSCAR were assessed by quantitative Real-Time PCR. Data represent the mean \pm SD of samples in triplicates. \#, *, and ${ }^{* *}$ indicate $p$ values $<0.05,<0.01$, and $<0.001$, respectively, vs the control. (B) BMMs were transduced with either pMXIRES-EGFP (control) or NRROS retrovirus. Transduced BMMs were serum-starved for 6 hours and stimulated with RANKL for the indicated time-periods. Whole cell lysates were then harvested from the cultured cells and immunoblotted with the antibodies indicated.

oblast differentiation, we performed an ALP activity assay. When primary osteoblasts were cultured in osteogenic conditions for 3 days, ALP activity was dramatically enhanced (Fig. 2B). However, increase in ALP activity in the NRROS-infected osteoblasts was comparable to that in the control vectorinfected cells. We then evaluated the effect of NRROS on osteoblast function by mineralized nodule staining. As shown in Fig. $2 \mathrm{C}$, bone nodule formation induced by osteogenic factors was indistinguishable in both the control vector- and NRROSinfected osteoblasts. Therefore, these results indicate that NRROS affects neither osteoblast differentiation nor function.

\section{NRROS negatively regulates osteoclast differentiation by} inhibiting the RANKL-mediated NF-KB pathway

To elucidate further the inhibitory role of NRROS in osteoclast differentiation, we sought to identify the osteoclastic genes that are inhibited upon NRROS overexpression. RANKL increased the expression of transcription factors including c-Fos and NFATc1 that are required for osteoclast differentiation, as well as osteoclast marker genes such as TRAP and OSCAR in the control vector-infected BMMs (Fig. 3A). Moreover, overexpres- sion of NRROS attenuated the expression of all the osteoclastic genes induced by RANKL (Fig. 3A). These results confirm that NRROS overexpression in BMMs inhibits RANKL-induced osteoclast differentiation. Moving forth, RANKL activates several early signaling pathways such as JNK, p38, ERK, and NF- $\mathrm{kB}$ that are essential to osteoclast differentiation. Since NRROS inhibited RANKL-induced expression of the osteoclastassociated genes, we next examined whether it also inhibits the RANKL-mediated early signaling pathways. We observed that overexpression of NRROS in BMMs strongly inhibited RANKL-induced NF- $\kappa B$ activation by blocking the degradation of I-KB proteins (Fig. 3B). Additionally, it slightly inhibited the RANKL-induced activation of all the MAPKs tested, except JNK (Fig. 3B). Therefore, these data collectively suggest that inhibition of the RANKL-induced signaling cascades (particularly that of NF- $\mathrm{KB}$ ) by NRROS attenuates osteoclastogenesis as well as osteoclastic gene expressions.

\section{NRROS represses NOX1 and NOX2 expressions} It is known that NRROS is a negative regulator of ROS production in phagocytes. To understand the underlying mechanism, 
we investigated the effect of NRROS on RANKL-mediated ROS production and the expressions of the NOX isozymes. As expected, overexpression of NRROS reduced the levels of intracellular ROS in osteoclasts, compared to that in control cells (Fig. 4A). According to our observations, NOX1 and NOX2, but not NOX3 and NOX4 are expressed in osteoclast precursor cells (Fig. 4B). Further, we cotransfected Plat E cells with NRROS and NOX isozymes to determine the effect of NRROS on the expressions of NOX1 and NOX2. This led to dramatic decrease in the protein expressions of both NOX1 and NOX2 (Fig. 4C). In addition, immunoprecipitation assays showed that both NOX1 and NOX2 directly interact with NRROS (Fig. 4D). Taken dation of NOX1 and NOX2 through direct interaction, which in turn might suppress the RANKL-induced ROS production.

\section{Downregulation of NRROS promotes RANKL-induced os- teoclast differentiation}

Finally, we investigated NRROS's physiological role in osteoclasts using siRNAs directed against the regulator. Upon transfection with NRROS siRNA, its mRNA levels were significantly abrogated in BMMs (Fig. 5A). This downregulation also significantly enhanced the RANKL-induced osteoclast differentiation (Fig. 5B). Overall, our data collectively suggest that NRROS

i $n \quad$ d $\quad$ e $\quad$ e $\quad d$

\section{A}

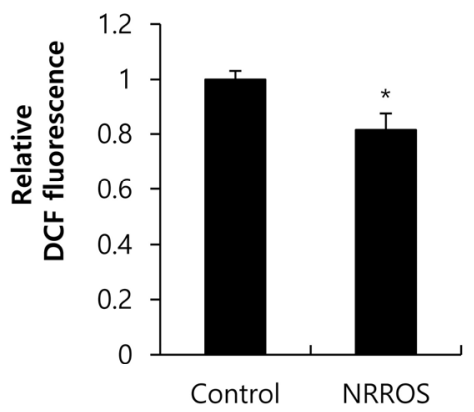

B
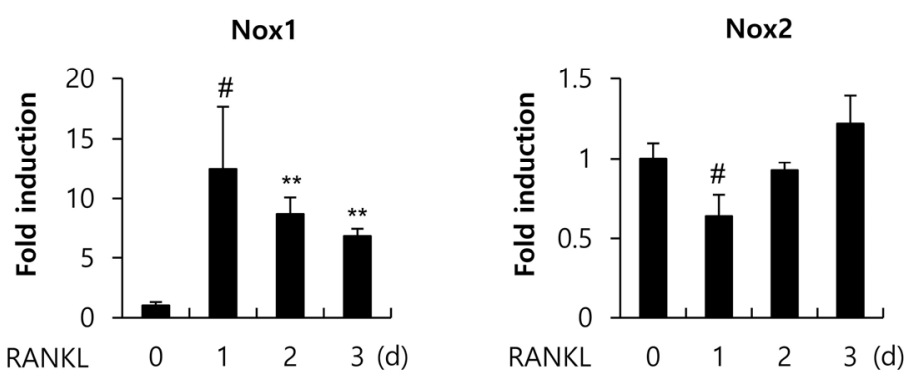

C

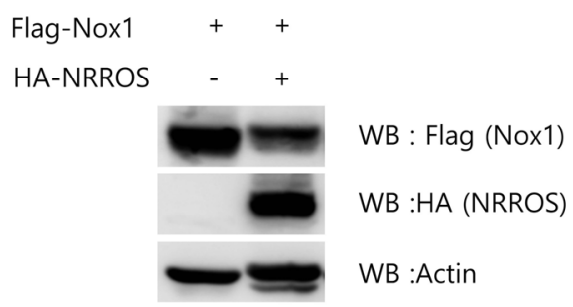

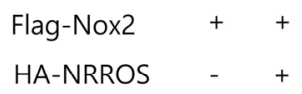

HA-NRROS - +

D

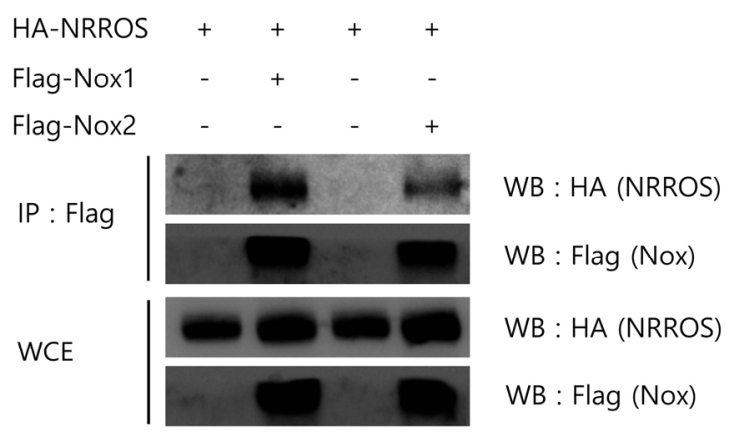

together, these results suggest that NRROS promotes degra-

Fig. 4. NRROS downregulates NOX1 and NOX2 expressions. (A) BMMs were transduced with either pMX-puro (control) or NRROS retrovirus, and then cultured in the presence of $\mathrm{M}$ CSF and RANKL for 3 days. Cultured cells were incubated with $10 \mu \mathrm{M}$ DCFH-DA for 30 min. DCF intensity was measured with an excitation wavelength of $488 \mathrm{~nm}$ and emission at $515-540 \mathrm{~nm} .{ }^{*}$ indicates $\mathrm{p}<0.01$ vs. the control. (B) BMMs were cultured with M-CSF and RANKL for the time-periods indicated. mRNA levels of NOX1 and NOX2 were assessed by quantitative Real-Time PCR. Data represent the mean \pm SD of samples in triplicates. (C) Plat E cells were cotransfected with either FLAG-NOX1 or FLAG-NOX2 along with either control vector or HA-NRROS. Whole cell lysates were harvested from the cultured cells and immunoblotted with the antibodies indicated. (D) Plat $\mathrm{E}$ cells were cotransfected with either HA-NRROS, control vector, FLAGNOX1, or FLAG-NOX2. Whole cell lysates were then harvested from the cultured cells and immunoprecipitated (IP) with anti-FLAG antibody. Next, IP samples or whole cell lysates were subjected to SDS PAGE and immunoblotted with the antibodies indicated. 
A

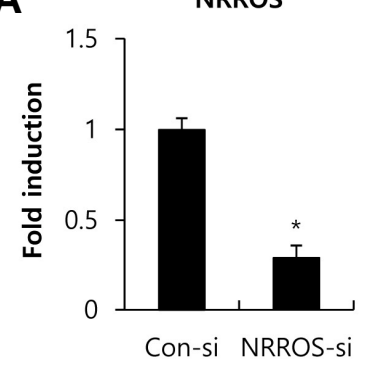

B

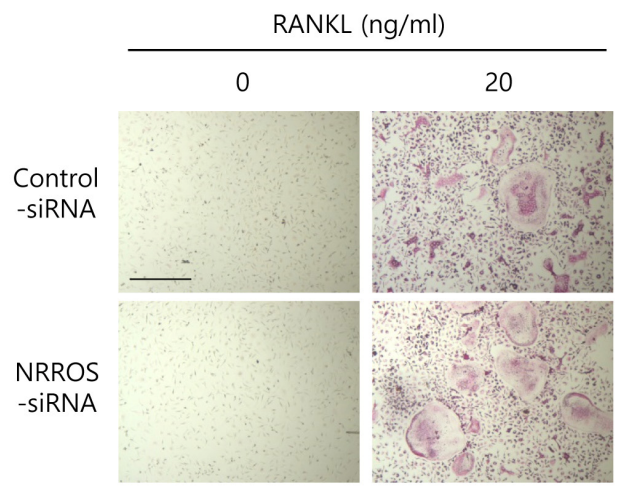

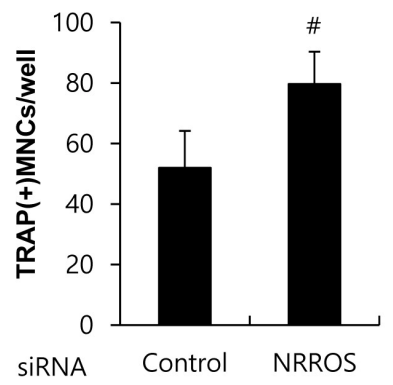

Fig. 5. Downregulation of NRROS enhances RANKL-induced osteoclastogenesis. (A, B) BMMs were transfected with either control or NRROS siRNAs. (A) mRNA levels of NRROS were assessed by quantitative Real-Time PCR. Data represent the mean $\pm S D$ of samples in triplicates. * indicates $p<0.01$ vs the control. (B) Transfected BMMs were cultured in presence of either M-CSF alone or together with RANKL for 3 days. Cultured cells were then stained for TRAP (left panel). The numbers of TRAP+ MNCs per well were counted (right panel). \# indicates $p<0.05$ vs the control. Bar, $100 \mu \mathrm{m}$. inhibits RANKL-mediated osteoclast differentiation.

\section{DISCUSSION}

In this study, we report for the first time the inhibitory role of NRROS in RANKL-induced osteoclast differentiation. We showed that overexpression of NRROS in BMMs attenuates RANKL-induced osteoclast differentiation (Fig. 1). Conversely, knockdown of NRROS by siRNA in BMMs significantly enhanced osteoclast formation (Fig. 5). Ectopic expression of NRROS in BMMs also inhibited the expressions of osteoclastspecific genes such as TRAP and OSCAR (Fig. 3).

Hematopoietic progenitor cells lacking c-Fos are unable to differentiate into osteoclasts, while mice lacking c-Fos, develop osteopetrosis (Grigoriadis et al., 1994; Johnson et al., 1992). This clearly indicates that c-Fos is indispensable to osteoclast differentiation both in vitro and in vivo. NF-кB p50/p52 double knockout mice also exhibit osteopetrotic bone phenotype (Yamashita et al., 2007). Yamashita et al. (2007) showed that RANKL-induced c-Fos expression and osteoclast differentiation were impaired in the NF- $\mathrm{KB}$ double knockout osteoclast precursors, and these defects were rescued by c-Fos overexpression. Therefore, it is inferred that NF- $\kappa B$ lies upstream to c-Fos in the RANKL-induced osteoclast differentiation. In this study, we also observed that NRROS overexpression inhibits c-Fos induction as well as NF- $\kappa B$ activation mediated by RANKL (Fig. $3 \mathrm{~A}$ and $3 \mathrm{~B})$. This inactivation of NF-kB in osteoclast precursors might prevent c-Fos induction, which in turn, could inhibit RANKLinduced osteoclast differentiation.

ROS are required for optimal osteoclast differentiation and function (Lee et al., 2005). Membrane-localized NADPH oxidases (NOXs) are involved in ROS generation in both phagocytic and nonphagocytic cells (Lambeth, 2004). Lee et al. (2005) reported that RANKL transiently increases intracellular ROS production while NOX inhibitors block this ROS genera- tion and osteoclastogenesis. These results establish that NOXs are required for RANKL-induced ROS generation and osteoclast differentiation. NRROS is known as a negative regulator of ROS production in phagocytes (Noubade et al., 2014). Moreover, this function of NRROS is associated with degradation of NOX2 through the ER-associated degradation pathway (Noubade et al., 2014). Our results showed that NRROS reduces the RANKL-induced ROS production and degrades the NOX1 and NOX2 proteins by direct interaction (Fig. 4). Taken together, these results support the hypothesis that NOX1 and NOX2 degradation by NRROS overexpression in osteoclast precursor cells inhibits RANKL-induced ROS generation and osteoclast differentiation.

ROS activates MAPKs under the control of several stimuli including growth factors and cytokines (Lee et al., 2005). ROS also activates MAPKs in osteoclast precursor cells, which might regulate osteoclast differentiation. However, NRROS overexpression had no effect on RANKL-induced JNK activation in this study, and only slightly inhibited p38 and ERK activation (Fig. 3B). Zhou et al. recently reported that Steap4, a member of the six transmembrane epithelial antigen of prostate (Steap) family proteins, plays a critical role in osteoclast differentiation by regulating ROS levels. They demonstrated that a decrease in ROS levels caused by Steap4 deficiency regulates osteoclast differentiation by inhibiting CAMP response element bonding protein (CREB), rather than by MAPK activation (Zhou et al., 2013). Interestingly, Lee et al. showed that silencing of NOX1 in osteoclast precursor cells blocks RANKL-mediated activation of JNK or p38, but does not affect ERK activation (Lee et al., 2005). It is possible that the differential effect of ROS-blocking on RANKL-mediated MAPK activation is due to the distinct mechanisms by which different ROS blockers inhibit its generation. Decrease in ROS levels by NOX1 downregulation might modulate RANKL-induced JNK or p38 activation. On the other hand, decrease in mitochondria-derived ROS by Steap4 might 
repress RANKL-induced CREB activation. Therefore, decrease in ROS levels, upon degradation of both NOX1 and NOX2, might inhibit RANKL-induced p38 or ERK activation. Only minor effects of NOX2 on ROS production and osteoclastogenesis, induced by RANKL, have been reported. Nevertheless, we cannot rule out the possibility that NOX2 degradation by NRROS contributes to RANKL-mediated ROS inhibition, and p38 or ERK activation.

In summary, the present study suggests a novel role for NRROS in osteoclast differentiation, wherein it acts as a negative regulator through the inhibition of two distinct signaling pathways: RANKL-mediated NF- $\mathrm{KB}$ activation and NOXdependent ROS generation.

\section{ACKNOWLEDGMENTS}

This work was supported by the Basic Science Research Program through the National Research Foundation of Korea (NRF) funded by the Ministry of Science, ICT, and Future Planning (2014R1A2A2A01003966, NRF-2012R1A1A3004341, and NRF-2013R1A1A2057964).

\section{REFERENCES}

Boyle, W.J., Simonet, W.S., and Lacey, D.L. (2003). Osteoclast differentiation and activation. Nature 423, 337-342.

Grigoriadis, A.E., Wang, Z.Q., Cecchini, M.G., Hofstetter, W., Felix, R., Fleisch, H.A., and Wagner, E.F. (1994). c-Fos: a key regulator of osteoclast-macrophage lineage determination and bone remodeling. Science (New York, N.Y.) 266, 443-448.

He, Y., Staser, K., Rhodes, S.D., Liu, Y., Wu, X., Park, S.J., Yuan, J., Yang, X., Li, X., Jiang, L., et al. (2011). Erk1 positively regulates osteoclast differentiation and bone resorptive activity. PloS one 6 , e24780.

Johnson, R.S., Spiegelman, B.M., and Papaioannou, V. (1992). Pleiotropic effects of a null mutation in the c-fos proto-oncogene. Cell 71, 577-586.

Kamiya, N. (2012). The role of BMPs in bone anabolism and their potential targets SOST and DKK1. Curr. Mol. Pharmacol. 5, 153163

Kim, J.H., and Kim, N. (2014). Regulation of NFATc1 in Osteoclast Differentiation. J. Bone Metabol. 21, 233-241.

Kim, K., Kim, J.H., Lee, J., Jin, H.M., Lee, S.H., Fisher, D.E., Kook, H., Kim, K.K., Choi, Y., and Kim, N. (2005). Nuclear factor of activated T cells $\mathrm{c} 1$ induces osteoclast-associated receptor gene expression during tumor necrosis factor-related activationinduced cytokine-mediated osteoclastogenesis. J. Biol. Chem.
280, 35209-35216.

Kim, K., Kim, J.H., Lee, J., Jin, H.M., Kook, H., Kim, K.K., Lee, S.Y., and Kim, N. (2007). MafB negatively regulates RANKLmediated osteoclast differentiation. Blood 109, 3253-3259.

Kim, K., Lee, S.H., Ha Kim, J., Choi, Y., and Kim, N. (2008). NFATc1 induces osteoclast fusion via up-regulation of Atp6v0d2 and the dendritic cell-specific transmembrane protein (DC-STAMP). Mol. Endocrinol. 22, 176-185.

Kim, K., Kim, J.H., Kim, I., Lee, J., Seong, S., Park, Y.W., and Kim, N. (2015). MicroRNA-26a regulates RANKL-induced osteoclast formation. Mol. Cells 38, 75-80.

Lambeth, J.D. (2004). NOX enzymes and the biology of reactive oxygen. Nat. Rev. Immunol. 4, 181-189.

Lee, N.K., Choi, Y.G., Baik, J.Y., Han, S.Y., Jeong, D.W., Bae, Y.S., Kim, N., and Lee, S.Y. (2005). A crucial role for reactive oxygen species in RANKL-induced osteoclast differentiation. Blood 106 , 852-859.

Moon, J.B., Kim, J.H., Kim, K., Youn, B.U., Ko, A., Lee, S.Y., and Kim, N. (2012). Akt induces osteoclast differentiation through regulating the GSK3beta/NFATc1 signaling cascade. J. Immunol. 188, 163-169.

Noubade, R., Wong, K., Ota, N., Rutz, S., Eidenschenk, C., Valdez, P.A., Ding, J., Peng, I., Sebrell, A., Caplazi, P., et al. (2014) NRROS negatively regulates reactive oxygen species during host defence and autoimmunity. Nature 509, 235-239.

Rho, J., Takami, M., and Choi, Y. (2004). Osteoimmunology: interactions of the immune and skeletal systems. Mol. Cells 17, $1-9$.

Sasaki, H., Yamamoto, H., Tominaga, K., Masuda, K., Kawai, T., Teshima-Kondo, S., and Rokutan, K. (2009). NADPH oxidasederived reactive oxygen species are essential for differentiation of a mouse macrophage cell line (RAW264.7) into osteoclasts. J. Med. Invest. 56, 33-41.

Walsh, M.C., Kim, N., Kadono, Y., Rho, J., Lee, S.Y., Lorenzo, J., and Choi, Y. (2006). Osteoimmunology: interplay between the immune system and bone metabolism. Annu. Rev. Immunol. 24 33-63.

Yamashita, T., Yao, Z., Li, F., Zhang, Q., Badell, I.R., Schwarz, E.M., Takeshita, S., Wagner, E.F., Noda, M., Matsuo, K., et al. (2007). NF-kappaB p50 and p52 regulate receptor activator of NFkappaB ligand (RANKL) and tumor necrosis factor-induced osteoclast precursor differentiation by activating c-Fos and NFATc1. J. Biol. Chem. 282, 18245-18253.

Zhou, J., Ye, S., Fujiwara, T., Manolagas, S.C., and Zhao, H. (2013) Steap4 plays a critical role in osteoclastogenesis in vitro by regulating cellular iron/reactive oxygen species (ROS) levels and cAMP response element-binding protein (CREB) activation. J. Biol. Chem. 288, 30064-30074. 